African Crop Science Journal by African Crop Science Society is licensed under a Creative Commons Attribution 3.0 Uganda License. Based on a work at www.ajol.info/ and www.bioline.org.br/cs DOI: http://dx.doi.org/10.4314/acsj.v25i4.4

\title{
NORMES TECHNIQUES ET PRATIQUES LOCALES DES PRODUCTEURS DANS LES PERIMETRES RIZICOLES IRRIGUES DE TOULA ET DE BONFEBA AU NIGER
}

\author{
I. MOSSI MAÏGA, A. ALOU HIMADOU ${ }^{1}$, A. HAOUGUI, A. SOULEYMANE et G. IBRO \\ Institut National de la Recherche Agronomique du Niger (INRAN), BP : 429 Niamey, Niger \\ ${ }^{1}$ Université de Tillabéri (UTI), BP : 175 Tillabéri, Niger \\ Auteur correspondant: imossim@yahoo.fr
}

(Received 6 August, 2017; accepted 13 October, 2017)

\begin{abstract}
RÉSUMÉ
Au Niger, la riziculture contribue pour beaucoup dans la sécurité alimentaire des populations. Les différents investissements effectués par le pays ont permis de promouvoir la riziculture irriguée dans la vallée du fleuve avec deux récoltes de riz par an et donc d'augmenter significativement les productions. Mais, force et de constater que les normes techniques censées être en application sur les périmètres rizicoles irrigués ont subi des changements au fil des années réduisant, ainsi, les performances espérées de cette riziculture moderne. La présente étude avait pour objectif d' analyser l'évolution des normes techniques de la production du riz et les pratiques locales dans les périmètres irrigués. L'étude s'est déroulée sur deux périmètres rizicoles irrigués au Niger et s'est basée sur des enquêtes auprès d'informateurs clés. Les enquêtes se sont basées sur un guide-questionnaire où il s'agit pour l'informateur-clé de répondre à différentes questions en rapport avec les pratiques agricoles liées à la culture du riz dans les périmètres rizicoles irrigués. L'analyse des données a été effectuée en utilisant les logiciels Excel, $\mathrm{R}$ Commender et le logiciel Nvivo. Les résultats obtenus ont montré qu'il y a une grande diversité entre les normes techniques et les pratiques sur le terrain. En plus des variétés homologuées, le choix des producteurs a porté aussi sur les variétés appelées communautaires alors que les règles l'ont interdit sur les périmètres irrigués. Les repiquages n'ont pas respecté la norme qui était de 25 poquets par $\mathrm{m}^{2}$. Les doses d'herbicide apportées par les producteurs ont varié de 80 à $240 \mathrm{~g} \mathrm{ha}^{-1}$ alors que la dose recommandée était de $80 \mathrm{~g} \mathrm{ha}^{-1}$. La fertilisation minérale étaitloin des normes prescrites non seulement en termes de quantité mais aussi en termes de fréquence d'application. Ces sous performances sont dues à l'inefficience de l'encadrement technique qui n'arrive pas à toucher le plus grand nombre de producteurs. L'encadrement technique manque de moyens tant matériel que technique et se base encore sur des méthodes traditionnelles pour diffuser le conseil agricole. Les méthodes appliquées aujourd'hui sont dépassées. Il serait donc nécessaire que les méthodes de diffusion du conseil agricole soient révisées. L'utilisation des nouvelles technologies de l'information et de la communication peut être une bonne opportunité pour une diffusion efficace du conseil agricole.
\end{abstract}

Mots Clés: Périmètre rizicole irrigué, normes techniques, riziculture

\begin{abstract}
In Niger, rice cultivation contributes significantly to the food security of populations. The various investments made by the country have made it possible to promote irrigated rice growing in the valley of the river with two crops of rice per year and thus to increase production significantly But it is clear that the technical standards that are supposed to be applied to irrigated rice areas have undergone changes over the years, thus reducing the
\end{abstract}


expected performance of this modern rice crop. The objective of this study was to analyse the evolution of technical standards of rice production and local practices in irrigated areas. The study was carried out on two irrigated rice-producing sites in Niger and was based on survey involving key informants. The results showed that there was a great diversity between technical standards and practices in the fields. In addition to the approved varieties, the choice of producers also covered Community varieties, whereas this practice is prohibited on irrigated areas. Transplanting did not meet the standard of 25 pockets per $\mathrm{m}^{2}$. The herbicide doses used by the producers varied from 80 to $240 \mathrm{~g} \mathrm{ha}^{-1}$; while the recommended one was $80 \mathrm{~g} \mathrm{ha}^{-1}$. Mineral fertilisation was different from the prescribed standards; not only in terms of quantity but also in terms of application frequency. These under performances are due to the inefficiency of technical management which does not reach the greatest number of producers. Technical supervision lacks technical and material resources and is still based on traditional methods for disseminating agricultural advice. The methods applied today are outdated. It would, therefore, be necessary to revise the farm council's dissemination methods.

Key Words : Rice cultivation, rice irrigated fields, standards technical

\section{INTRODUCTION}

Le Niger est un pays sahélien très souvent confronté à des déficits alimentaires à cause des aléas climatiques (Sido et al., 2015). Comme dans plusieurs pays du Sahel, la maîtrise de l'eau, à travers l'irrigation a été promue pour augmenter et diversifier les productions agricoles (Mossi Maïga, 2009). Pour atteindre les objectifs de la sécurité alimentaire recherchée, la maîtrise de l'eau a été accompagnée de celle des techniques culturales à travers un système d'appui conseil performant (MAG., 2015). Le cas des périmètres irrigués est le plus illustratif car des conditions spécifiques ont été créées, aussi bien en amont qu'en aval aux fins d'une forte intensification. La création d'un office chargé de l'aménagement des périmètres irrigués et l'encadrement rapproché des producteurs, la mise en place de services en charge de l'approvisionnement en intrants agricoles subventionnés, de la transformation et de la commercialisation des produits, de la facilitation de crédits auprès d'institutions financières sont, entre autres, des conditions pour garantir durablement les productions irriguées (Mossi Maïga, 2009). Avec le temps les normes techniques de production sur les périmètres irrigués ont subi des transformations. L'itinéraire technique proposé : mise en boue-travail du sol-semis en pépinière-fumure de fond-repiquage- désherbage 1-fumure de couverture 1désherbage 2-fumure de couverture 2-récolte, avec les apports d'eau tout au long du cycle cultural n'est plus respecté. Il en est de même pour les quantités de fertilisant et de semences, les périodes de fertilisation et de récolte. Le paquet technique proposé par l'encadrement technique au Niger et qui se résume à $50 \mathrm{~kg}$ de semence de riz pour la production de plants pour le repiquage d'un hectare de riz, $400 \mathrm{~kg}$ ha-1 d'engrais en deux apports selon des périodes bien déterminées, le repiquage des plants de 30 jours pour la culture du riz de saison d'hivernage et de 45 jours pour la culture du riz de saison sèche, le repiquage d'un plan de riz par poquet à une densité de 250000 plants ha ${ }^{-1}$, drainage de la parcelle de riz à 15 à 30 jours avant la récolte (Ekoye et al., 1996) n'est pas non plus respecté. Tous ces nonrespects se répercutent sur les rendements, les coûts de production, l'intensité culturale (Poussin et al., 2002). Si les espérances portées sur la riziculture irriguée ont tablé sur des rendements de 8 à 12 tonnes par hectare, faute a été de reconnaitre que ces rendements sont en moyenne inférieurs à 5 tonnes par hectare, sont variables et aussi instables. En cause, le manque de maîtrise des techniques au niveau de l'irrigation, de la fertilisation et de l'enherbement (Poussin, 1994). Les producteurs dans les périmètres rizicoles irrigués se sont réapproprié les normes, que chacun cherche à appliquer plus ou moins 
efficacement. La présente étude avait pour objectif d'analyser l'évolution des normes techniques de la production du riz en pratiques locales dans les périmètres irrigués.

\section{MATÉRIEL ET MÉTHODE}

Les enquêtes se sont déroulées sur les périmètres irrigués rizicoles de Toula et de Bonféba dans le département de Tillabéri au Niger (coordonnées géographiques $\left.14^{\circ} 11^{\prime} 37.83 \mathrm{~N} / 1^{\circ} 28^{\prime} 13.78 \mathrm{E}\right)$. Sur ces deux sites, l'irrigation est bien maîtrisée car elle est assurée grâce à des pompes immergées et des réseaux d'irrigation et de drainage pour la distribution de l'eau entre les producteurs et le drainage des eaux. Ces périmètres sont exploités par des producteurs organisés en coopératives dont les dirigeants sont élus en assemblées générales. Les producteurs sont encadrés par un technicien permanent, appelé directeur de périmètre, qui est un agent mis à disposition par l'Etat à travers l'office national des aménagements hydro-agricoles (ONAHA). Les caractéristiques techniques et sociales des deux périmètres sont consignées dans le Tableau 1.

Les enquêtes diagnostics ont concerné 20 producteurs (numérotés de 1 à 20) considérées comme des informateurs clés (AfricaRice, 2013). Ils ont été choisis en se basant sur leur expérience et leur connaissance des systèmes de production agricole et du riz dans les villages et sur les périmètres irrigués. Comme le montre le Tableau 2, parmi eux il y a les présidents des coopératives, les chefs de village, les producteurs, les agents techniques des périmètres.

Les enquêtes se sont basées sur un guidequestionnaire (AfricaRice, 2013) où il s'agit pour l'informateur-clé de répondre à différentes questions en rapport avec les pratiques agricoles liées à la culture du riz dans les périmètres irrigués rizicoles.

L'analyse des données a été effectuée en utilisant les logiciels Excel et $R$ Commender ainsi que le logiciel Nvivo qui permet de faire des analyses textuelles. 
TABLEAU 2. Caractéristiques de l'échantillon d'étude

\begin{tabular}{|c|c|c|c|c|c|c|c|}
\hline Informateur & Périmètre & Activité principale & Activité secondaire & âge & Niveau d'éducation & Activités non-agricole & $\begin{array}{r}\text { Superficie } \\
\text { rizicole (ha) }\end{array}$ \\
\hline 1 & Bonféba & Producteur & & 80 & alphabétisation & & 0,52 \\
\hline 2 & Bonféba & cultivateur & & 80 & l'école coranique & & 0,48 \\
\hline 3 & Bonféba & Cultivateur & Artisan & 39 & classe de 6eme & Artisan & 0,58 \\
\hline 4 & Bonféba & directeur de périmètre & Producteur & 59 & BEPC & Producteur & 1 \\
\hline 5 & Bonféba & cultivateur & & 45 & & & 0,7 \\
\hline 6 & Bonféba & cultivateur & Commerçant & 58 & alphabétisation & Commerçant & 2 \\
\hline 7 & Bonféba & agriculteur & Conseiller municipal & 52 & & $\begin{array}{l}\text { Transporteur et Conseiller } \\
\text { municipal }\end{array}$ & 0,75 \\
\hline 8 & Bonféba & cultivateur & & 49 & collège : $3^{\text {ème }}$ & & 0,89 \\
\hline 9 & Bonféba & comptable & Producteur & 45 & Comptable & Producteur & 1,5 \\
\hline 10 & Bonféba & agriculteur & & 44 & collège : $5^{\text {ème }}$ & & 0,3 \\
\hline 11 & Bonféba & riziculture & Pompiste sur le périmètre & 30 & collège $: 3^{\text {ème }}$ & Pompiste sur le périmètre & 0,5 \\
\hline 12 & Toula & cultivateur & & 34 & Ecole coranique & & 0,85 \\
\hline 13 & Toula & cultivateur & & 38 & $\mathrm{CM} 2$ & & 0,15 \\
\hline 14 & Toula & cultivateur & Pompiste sur le périmètre & 55 & $\begin{array}{l}\text { Ecole coranique et } \\
\text { alphabétisation }\end{array}$ & Pompiste sur le périmètre & 0,25 \\
\hline 15 & Toula & cultivateur & & 55 & Ecole coranique & Chef du village & 0,25 \\
\hline 16 & Toula & cultivateur & comptable & 57 & Ecole primaire et coranique & & 0,29 \\
\hline 17 & Toula & cultivateur & commerçant & 47 & alphabétisation & & 0,64 \\
\hline 18 & Toula & directeur de périmètre & Producteur & 63 & collège $: 3^{3 \text { ème }}$ & DEP & 0,55 \\
\hline 19 & Toula & cultivateur & & 37 & & & 0,48 \\
\hline 20 & Toula & cultivateur & & 45 & Ecole coranique & & 1,5 \\
\hline
\end{tabular}




\section{RÉSULTATS}

Mise en place de la culture du riz dans les périmètres irrigués de Bonféba et de Toula Le choix des variétés. Les producteurs des périmètres irrigués de Toula et de Bonféba ont énuméré plusieurs variétés comme le montre le (Tableau 3). Certaines de ces variétés sont soit encore cultivées sur les périmètres, soit abandonnées depuis longtemps. Parmi celles encore en culture, on note la variété IR1529, la Gambiaka et la Waihidjo et la Karidjikoyo. Les deux premières sont des variétés de riz introduites formellement à partir de la recherche et du développement. Cependant, seule la variété IR1529 semble être bien connue dans ses différentes caractéristiques car ayant été introduite de longue date sur les périmètres. Les variétés Waihidjo et Karidjikoyo sont des variétés issues de sélections massales effectuées par les producteurs eux-mêmes et qui leur ont donné des noms locaux.

Ces variétés sont différemment implantées sur les périmètres. Sur le périmètre de Toula, le premier choix des informateur-clés s'est porté sur la variété IR1529 à $89 \%$, et le deuxième choix sur Waihidjo (44\%), et Gambiaka (15\%). Sur le périmètre de Bonféba, le premier choix s'est porté sur la Gambiaka $(36 \%)$ et IR1529 (18\%). Le deuxième choix s'est porté sur Waihidjo à (27\%) etIR1529 $(18 \%)$.

Les producteurs semblent apprécier ces variétés pour le rendement, le goût, la résistance au stress hydrique, la résistance aux oiseaux, le tallage. Le Tableau 4, résume les appréciations des producteurs vis-à-vis de ces variétés.

Mise en place des pépinières sur les périmètres irrigués de Toula et de Bonféba. Les semences utilisées pour les pépinières sont multipliées par des producteurs accrédités, choisis parmi les meilleurs.

Pour les semis, les semences sont distribuées aux producteurs au prorata des superficies cultivées.

TABLEAU 3. Variétés de riz énumérées par les producteurs sur les périmètres irrigués de Toula et de Bonféba

\begin{tabular}{|c|c|c|c|c|c|c|}
\hline Informateurs & Périmètre & Variété 1 & Variété 2 & Variété 3 & Variété 4 & Variété 5 \\
\hline 1 & Toula & IR1529 & Gambiaka & & & \\
\hline 2 & Toula & IR1529 & Waihidjo & & & \\
\hline 3 & Toula & IR1529 & Waihidjo & Mama & Lokki & \\
\hline 4 & Toula & IR1529 & Waihidjo & Gambiaka & Wita 8 & wita 9 \\
\hline 5 & Toula & IR1529 & Gambiaka & Waihidjo & & \\
\hline 6 & Toula & Karidjikoyo & IR1529 & BG 90-2 & Waihidjo & Gambiaka \\
\hline 7 & Toula & IR1529 & Waihidjo & Mama & Lokki & \\
\hline 8 & Toula & IR1529 & Gambiaka & & & \\
\hline 9 & Toula & IR1529 & Wita 8 & Waihidjo & Gakonouizo & \\
\hline 10 & Bonféba & Alewa & Somba & Gambiaka & & \\
\hline 11 & Bonféba & Somba & Waihidjo & & & \\
\hline 12 & Bonféba & IR1529 & & & & \\
\hline 13 & Bonféba & Gambiaka & Karidjikoyo & & & \\
\hline 14 & Bonféba & Karidjikoyo & Wita 8 & wita 9 & Waihidjo & \\
\hline 15 & Bonféba & Gambiaka & IR1529 & & & \\
\hline 16 & Bonféba & Waihidjo & & & & \\
\hline 17 & Bonféba & IR1529 & & & & \\
\hline 18 & Bonféba & Somba & Waihidjo & Gambiaka & & \\
\hline 19 & Bonféba & Gambiaka & Waihidjo & Somba & & \\
\hline 20 & Bonféba & Gambiaka & IR1529 & & & \\
\hline
\end{tabular}




\begin{tabular}{|c|c|c|}
\hline Nom de la variété & Caractéristiques physiques & Cycle (mois) \\
\hline Gambiaka & Produit beaucoup, talle bien, très bon goût & 6 mois. \\
\hline IR1529 & Produit beaucoup, bon tallage, bon goût, bon taux au décorticage & 6 mois \\
\hline Ouéhidjo & $\begin{array}{l}\text { Produit beaucoup, rempli bien les sacs mais, elle donne plus de son que de graines, les sacs ne sont pas lourds, elle résiste bien } \\
\text { au stress hydrique }\end{array}$ & 6 mois \\
\hline Somba & $\begin{array}{l}\text { Produit bien, les sacs ne sont pas lourds, la quantité de son obtenu après décorticage est élevée, résiste bien au déficit hydrique } \\
\text { La taille est haute, talle bien, est exigeante en engrais. }\end{array}$ & 6 mois \\
\hline Wita 8 & $\begin{array}{l}\text { Présente les mêmes caractéristiques que Ouéhidjo mais elle produit mieux en saison d'hivernage. Elle ne supporte pas la } \\
\text { sécheresse }\end{array}$ & 5 à 6 mois \\
\hline Wita 9 & $\begin{array}{l}\text { Présente les mêmes caractéristiques que Ouéhidjo mais elle produit mieux en saison d'hivernage. Elle ne supporte pas la } \\
\text { sécheresse }\end{array}$ & 5 à 6 mois \\
\hline Kardjikoyo & $\begin{array}{l}\text { Est résistante à la sécheresse et aux oiseaux à cause des épines qui sont sur les épis. Elle est exigeante en eau, produit bien. Elle } \\
\text { est de grande taille }\end{array}$ & 5 à 6 mois \\
\hline Gakonouizo & $\begin{array}{l}\text { Haute taille (>1m), faible tallage, panicule dressée, pas bon goût (cause d'abandon), taux de décorticage élevé, le riz préparé ne } \\
\text { se conserve pas (cause d'abandon), bonne production }\end{array}$ & 6 mois \\
\hline Mama & irrigué & - \\
\hline Lok'ki & irrigué & - \\
\hline
\end{tabular}


Les pépinières sont collectives et les résultats des enquêtes font ressortir que les pratiques sont presque identiques dans les 2 périmètres. Les étapes suivantes ont caractérisé la mise en place des pépinières de riz: Les sacs de semences, généralement de $75 \mathrm{~kg}$, sont plongés dans l'eau pendant 48 heures si c'est la culture de riz de la saison sèche et 24 heures en culture de saison d'hivernage. Ensuite, ils sont sortis de l'eau et recouverts de paille pendant 24 heures. Le fait de recouvrir les sacs de paille permettait d'augmenter la température à l'intérieur du sac afin de faciliter la pré-germination des semences. Les semences pré-germées sont traitées avec un insecticide-fongicide (Thioral) puis distribuées aux producteurs qui les sèment à la volée dans les pépinières. Les pépinières sont ensuite traitées au Furadan (un insecticide-nématicide) afin de préserver les jeunes plants contre les insectes défoliateurs. Selon les producteurs, les plants de riz peuvent passer de 45 à 60 jours dans les pépinières avant d'être repiqués pendant la saison froide (décembre-février). Par contre, les plants passent moins de 25 jours en pépinières pendant l'hivernage (Mai à Novembre) où les températures semblent plus favorables à la germination des semences de riz.

Repiquage du riz sur les périmètres de Toula et de Bonféba. Le repiquage est la pratique adoptée sur l'ensemble des périmètres irrigués rizicoles du Niger. Il se fait après un séjour des plants de riz en pépinière comme déjà précisé ci-dessus. La particularité du repiquage dans ces périmètres réside dans le fait qu'il est effectué par des enfants qui se constituent en petits groupes de 5 à 10 en prestataires de service. Le nombre de plants repiqués est élevé et la densité de repiquage est parfois très différente de la norme proposée par l'encadrement technique à savoir 250000 poquets par hectare. Dans les réponses sur le repiquage, les producteurs affirment utiliser une corde pour mieux délimiter les lignes et matérialiser les poquets.

\section{Travaux d'entretien}

Lutte contre les adventices et les oiseaux. La lutte contre les adventices et les oiseaux constituent une des plus importantes tâches sur les périmètres irrigués. Les adventices les plus couramment rencontrées sont Echinochloacolona (L). Cyperusrotundus (L), Sphenoclea zeylanica (Gaertner), Oryza longistaminata (Chev and Roehr) et Ludwigiasp (L).

Contre ces adventices, les producteurs utilisent, dans un premier temps, un herbicide (le Londax) et ensuite, procèdent à des désherbages manuels car l'herbicide utilisé n'est pas efficace sur toutes les adventices. Parmi les adventices résistantes à l'herbicide, ils ont cité $O$. longistaminata (Chev and Roehr) qui n'est autre que le riz sauvage et celle qu'ils ont appelé E.colona $(\mathrm{L})$ qui est très difficile à arracher.

L'utilisation des herbicides est très importance dans les pratiques paysannes. L'herbicide le plus couramment utilisé est le Londax que les coopératives conditionnent par paquet de $25 \mathrm{~g}$ correspondant au traitement d'une parcelle de 0,25 ha. Les enquêtes ont montré que les doses apportées par parcelle ainsi que les périodes d'application étaient parfois différentes selon les producteurs. En effet, la dose recommandée est de $80 \mathrm{~g} \mathrm{ha}^{-1}$. Les doses appliquées allaient de $80 \mathrm{~kg} \mathrm{ha}^{-1}$, à $100 \mathrm{~kg} \mathrm{ha}^{-1}$ et jusqu'à $240 \mathrm{~kg} \mathrm{ha}^{-1}$. Quant aux périodes d'application, le produit est souvent apporté 3 à 15 jours après le repiquage alors que les fiches techniques ont préconisé d'appliquer le Londax lorsque les adventices sont au stade 2 à 3 feuilles correspondant, approximativement, à 15 à 20 jours après le semis ou le repiquage.

Contre les oiseaux, les producteurs n'ont d'autres méthodes que le gardiennage. Pour lutter contre les oiseaux granivores (Quelea quelea (L)), les producteurs font recours aux enfants qui gardent les parcelles de 6 heures à 18 heures durant toute la période de remplissage et de la maturation des grains. 
Certains producteurs prétendent que les variétés de riz Waihidjo et Kardjikoyo résistent aux oiseaux; le premier ayant des épillets recourbés vers le sol donc hors d'atteinte des oiseaux, le second ayant des grains aristés gênant les oiseaux dans leur prise de nourriture Fertilisation minérale dans les périmètres de Toula et de Bonféba.

La fertilisation se fait à deux niveaux dans les périmètres rizicoles : dans les pépinières qui sont collectives et sous la responsabilité des coopératives (Fig. 1) et dans les parcelles individuelles sous la seule gestion du producteur.

Les quantités d'engrais apportées dans les pépinières étaient presque identiques dans les deux périmètres. Elles sont en général, d'un sac de 50 kg d'engrais composé NPK (15-1515) en culture de saison d'hivernage et de deux sacs de $50 \mathrm{~kg}$ d'engrais dont un sac d'engrais composé NPK (15-15-15) et un sac d'urée. Les superficies des pépinières étant en moyenne de 0,25 ha, les doses moyennes d'engrais apportées par hectare en culture de saison sèche est de $122 \mathrm{~N} 30 \mathrm{P} 30 \mathrm{~K}$ et pour la saison d'hivernage de 30N30P30K.

Pour ce qui est de l'apport des engrais dans les parcelles individuelles, la Figure 1 montre la dispersion des quantités totales d'engrais apportées par les producteurs des périmètres de Bonféba et de Toula.

Les quantités médianes apportées étaient respectivement de $506 \mathrm{~kg} \mathrm{ha}^{-1}$ pour le périmètre de Bonféba et de $700 \mathrm{~kg} \mathrm{ha}^{-1}$ pour celui de Toula, tout type d'engrais confondus.

Ces quantités totales d'engrais étaient généralement réparties par les producteurs en trois apports sur la saison culturale. Pour le premier apport d'engrais, à l'exception de quelques producteurs $(11 \%$ pour Toula et $27 \%$ pour Bonféba) qui faisaient un mélange d'engrais NPK et Urée, l'ensemble des producteurs a apporté de l'engrais composé NPK (15-15-15). La Figure 2 montre la dispersion des premiers apports d'engrais faits par les exploitants et par périmètre.

Les quantités médianes d'engrais apportées au premier apport étaient de $200 \mathrm{~kg} \mathrm{ha}^{-1}$ pour chaque périmètre. Cependant, plus de $75 \%$ des producteurs ont apporté plus de $350 \mathrm{~kg} \mathrm{ha}^{-1}$ sur le périmètre de Bonféba et plus de $300 \mathrm{~kg}$ ha $^{-1}$ sur celui de Toula. De cette quantité totale d'engrais apporté, les valeurs médianes d'azote $(\mathrm{N})$, de phosphore $(\mathrm{P})$ et de Potassium $(\mathrm{K})$ étaient les mêmes à savoir $30 \mathrm{~kg} \mathrm{ha}^{-1}$. On notera cependant que plus de $75 \%$ des producteurs ont apporté plus d'azote comme l'a montré le (Tableau 5).

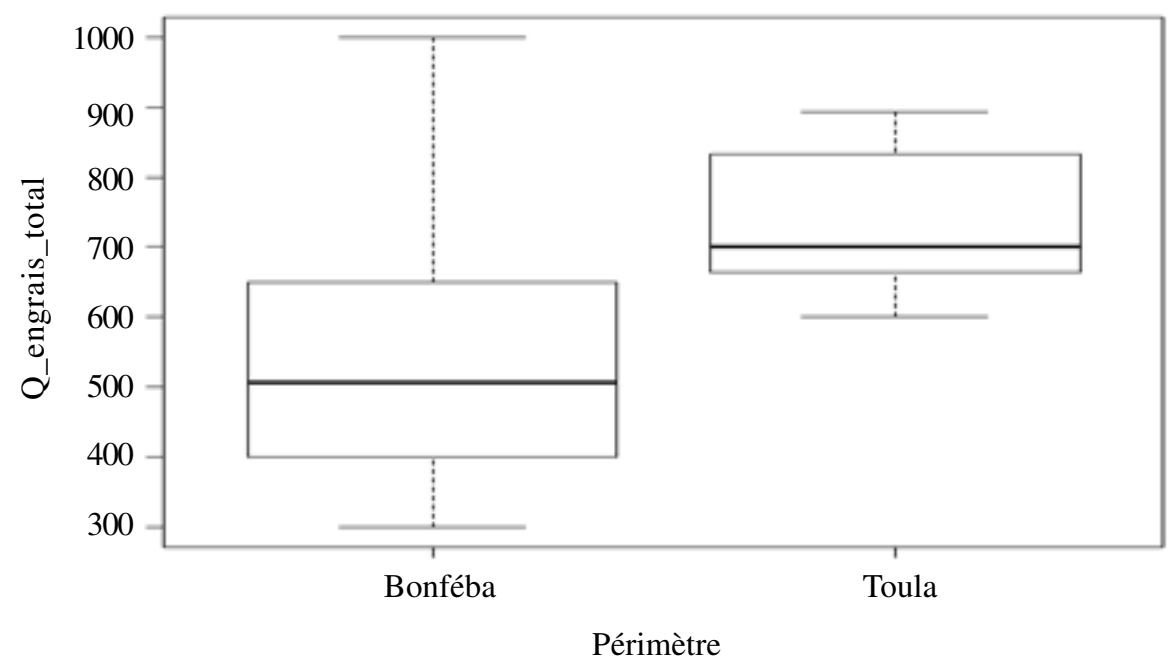

Figure 1. Dispersion des quantités totales d'engrais apporteés par exploitant et par périmètre. 


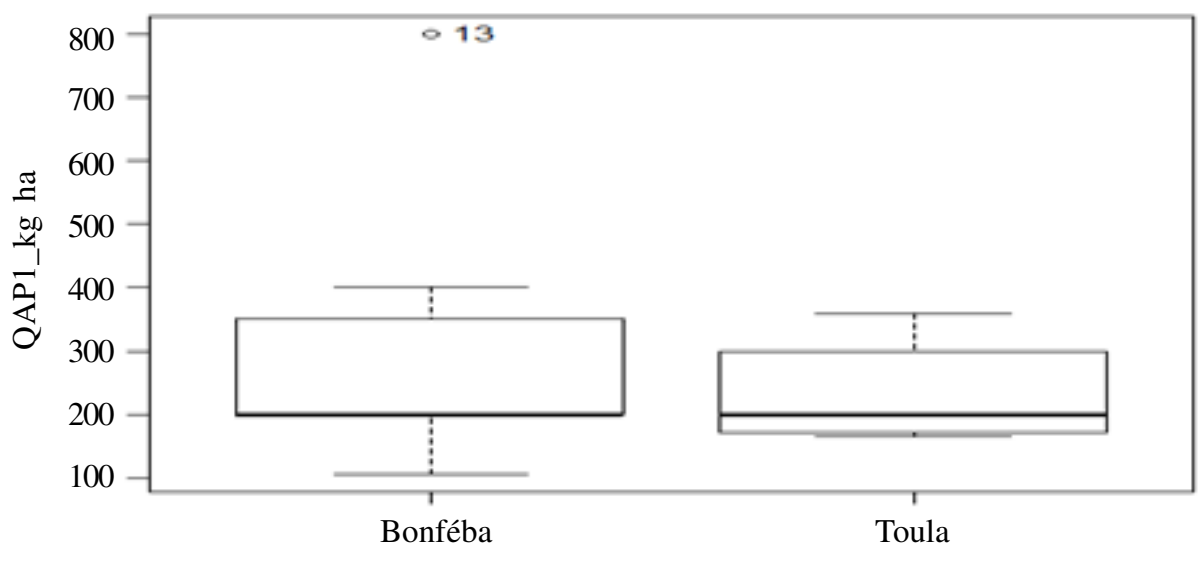

Périmètre

Figure 2. Dispersion des quantités d'engrais apportées au premier apport par périmètre et par producteur.

TABLEAU 5. Quantités d'éléments N, P, et K (valeurs médianes et quantile 0.75) apportées par les producteurs par périmètre

\begin{tabular}{|c|c|c|c|c|c|c|}
\hline & \multicolumn{2}{|c|}{ AP1_N (kg ha) } & \multicolumn{2}{|c|}{ AP1_P (kg ha) } & \multicolumn{2}{|c|}{ AP1_K (kg ha) } \\
\hline & médiane & quantile 0,75 & médiane & quantile 0,75 & médiane & quantile 0,75 \\
\hline Bonféba & 30 & 52,5 & 30 & 37,5 & 30 & 37,5 \\
\hline Toula & 30 & 45 & 30 & 41,55 & 30 & 41,55 \\
\hline
\end{tabular}

AP1- = premier apport d'élément fertilisant

Les premiers apports d'engrais ont été faits en moyenne 14 jours après le repiquage avec un maximum n'excédant pas 20 jours après le repiquage.

Les seconds apports d'engrais ont été faits, selon les médianes à 30 jours après le repiquage sur le périmètre de Bonféba et de 35 jours après le repiquage sur le périmètre de Toula. $75 \%$ des producteurs ont apporté la seconde fertilisation à 38,5 jours après le repiquage sur le périmètre de Bonféba et de 40 jours sur le périmètre de Toula.

Pour le second apport d'engrais, le choix du type d'engrais est porté par les producteurs de Toula sur l'urée ou sur le mélange urée et NPK (15-15-15). Quant aux producteurs du périmètre irrigué de Bonféba, ils ont porté leur choix à $72 \%$, sur l'urée au second apport. La
Figure 3 suivante a montré la dispersion des quantités d'engrais apportées par les producteurs par périmètre à la deuxième fertilisation.

On a constaté que la deuxième fertilisation d'engrais n'a pas dépassé $200 \mathrm{~kg} \mathrm{ha}^{-1}$ sur le périmètre de Bonféba où la valeur médiane est d'ailleurs de $200 \mathrm{~kg} \mathrm{ha}^{-1}$. Sur le périmètre de Bonféba, la valeur médiane des deuxièmes apports était de $278 \mathrm{~kg} \mathrm{ha}^{-1}$.

De cette quantité totale d'engrais apportée à la seconde fertilisation, l'élément azote $(\mathrm{N})$ a occupé une bonne place comme on peut le noter dans le (Tableau 6).

La troisième fertilisation s'est faite selon une médiane de 45 jours après le repiquage par les producteurs du périmètre de Bonféba et de 50 jours après le repiquage sur celui de 


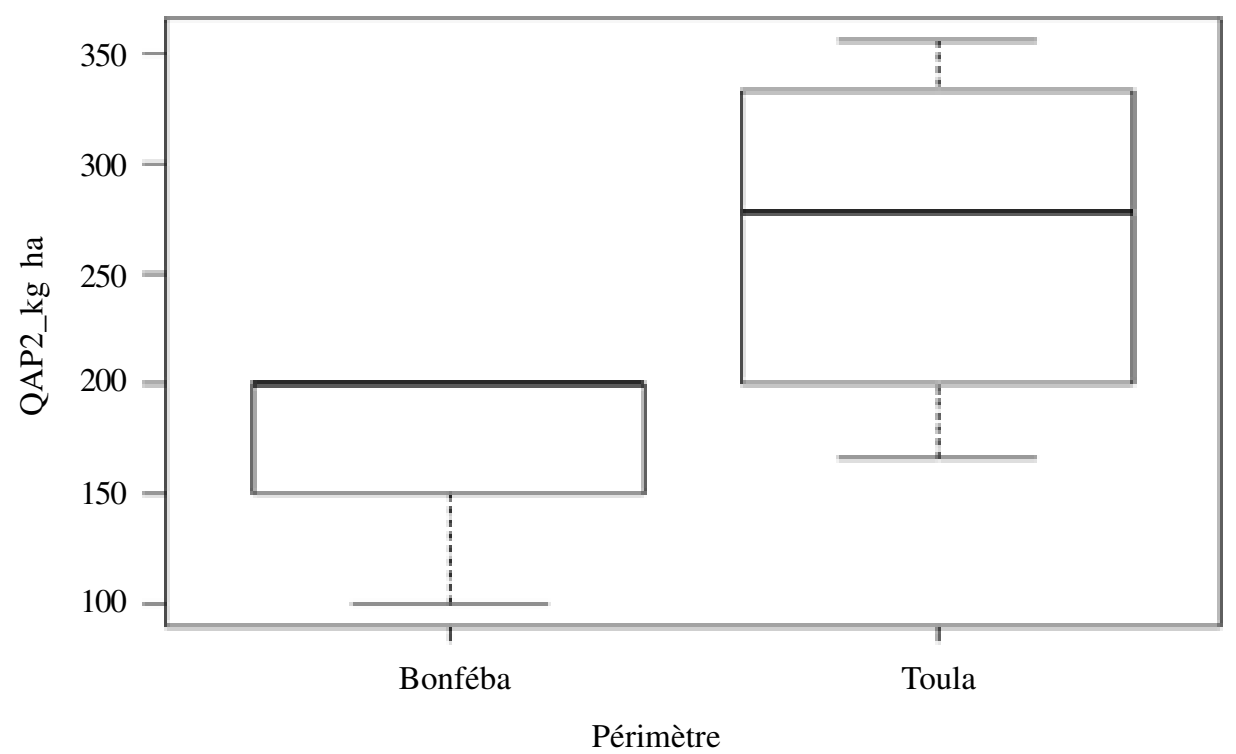

Figure 3. Dispersion des quantités d'engrais apportées au second apport par périmètre par les producteurs des périmètres.

TABLEAU 6. Quantités d'éléments N, P, et K (valeurs médianes et quantiles 0.75) apportés par les producteurs par périmètre

\begin{tabular}{|c|c|c|c|c|c|c|}
\hline & \multicolumn{2}{|c|}{ AP2_N (kg ha) } & \multicolumn{2}{|c|}{ AP2_P (kg ha) } & \multicolumn{2}{|c|}{ AP2_K (kg ha) } \\
\hline & médiane & quantile 0,75 & médiane & quantile 0,75 & médiane & quantile 0,75 \\
\hline Bonféba & 61 & 92 & 0 & 7,5 & 0 & 7,5 \\
\hline Toula & 70 & 100 & 25,8 & 30 & 24,9 & 30 \\
\hline
\end{tabular}

AP2- = Deuxième apport d'élément fertilisant

Toula. Plus de $75 \%$ l'ont fait à 50 jours après le repiquage à Bonféba et à 60 jours après le repiquage à Toula.

La troisième fertilisation était soit constituée d'urée soit de mélange d'urée et de NPK (15-15-15). Certains producteurs n'ont pas fait la troisième fertilisation. La Figure 4 suivante a montré la dispersion des quantités totales d'engrais apportées par les producteurs par périmètre. La quantité médiane d'engrais apportée à la troisième fertilisation par les producteurs du périmètre de Bonféba était de $100 \mathrm{~kg} \mathrm{ha}^{-1}$. Sur le périmètre de Toula la médiane était de $277 \mathrm{~kg} \mathrm{ha}^{-1}$. Près de $75 \%$ des producteurs de Bonféba ont apporté plus de $200 \mathrm{~kg} \mathrm{ha}^{-1}$ et ceux de Toula, ont appliqué environ $332 \mathrm{~kg} \mathrm{ha}^{-1}$.

Les troisièmes apports ont surtout constitués d'azote comme l'a montré le [Tableau 7 suivant.

\section{DISCUSSION}

Plusieurs enquêtes (MAG et KFW, 2015a, MAG et KFW, 2015b ; Mossi Maïga, 2009 ; CNI-B, 2009 ; CNI-B, 2010 ; Sido et Mossi 


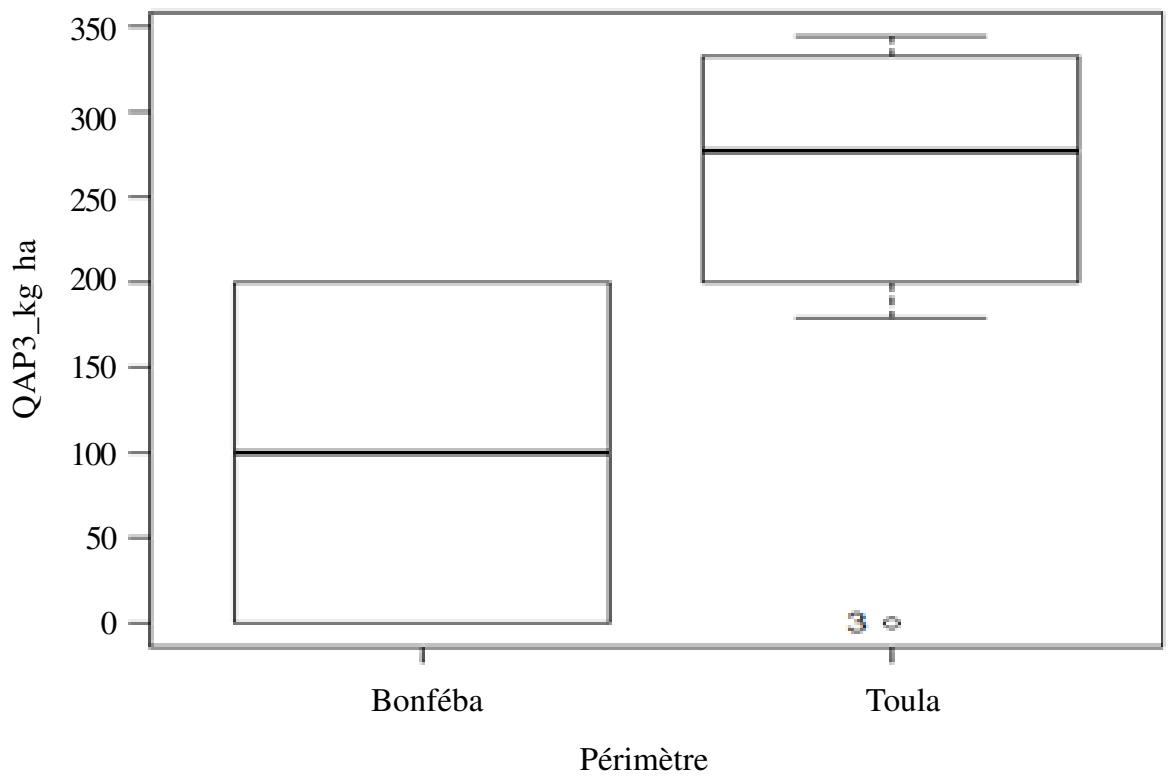

Figure 4. Dispersion des quantités d'engrais apportées au troisième apport par périmètre pour les producteurs des périmètres.

TABLEAU 7. Quantités d'éléments N, P et K (valeurs médianes et quantiles 0.75 ) apportés par les producteurs par périmètre

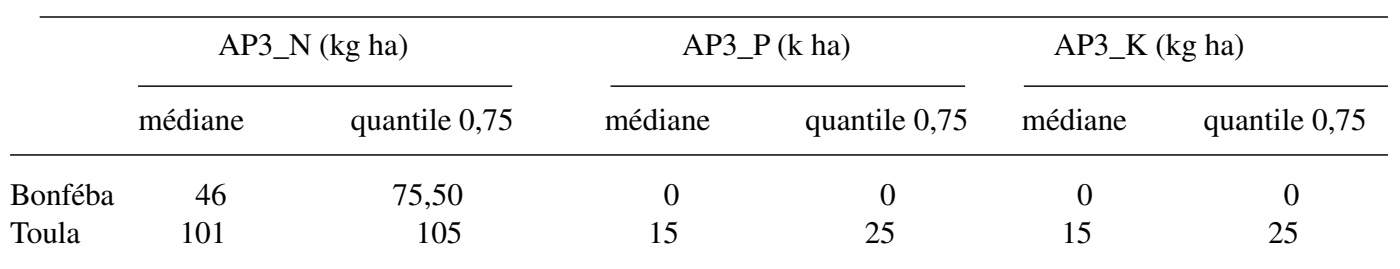

AP3- = troisième apport d'élément fertilisant

Maïga, 2003, CGERV, 2012) effectuées dans les périmètres irrigués ont montré une grande diversité des pratiques mises en œuvre par les producteurs. Ces périmètres irrigués ont pourtant été conçus selon une logique techniciste où, comme le disait Mossi Maïga (2009), le respect des normes devrait permettre d'aboutir à un certain niveau de production. La diversité dans les pratiques a été remarquée dans toutes les étapes de la culture du riz aussi bien dans la mise en place de la culture que dans les travaux d'entretien. Il ressort de cette étude que le choix des variétés ne semblait plus être du seul ressort de l'encadrement technique comme on pouvait le noter dans la multitude des variétés actuellement cultivées sur ces deux périmètres. A côté des variétés homologuées à savoir la Gambiaka et la IR1529, on trouve surtout des variétés appelées variétés communautaires. D'autres études menées au Niger ont fait les mêmes constats où les producteurs ont remplacé les variétés homologuées par d'autres variétés locales. C'est le cas sur les périmètres irrigués rizicoles de Gaya amont et Say 1 au Niger où les études (MAG et KFW, 2015a) ont montré que des variétés dites communautaires ont pris la place 
des variétés homologuées. Sur le périmètre de Gaya amont, il a été distingué des variétés comme Samo Sagoumi, Mali izo, AkilouAkilou, Fondia, Jumbo. Sur le périmètre de Say 1, c'était la variété locale Alewa qui prédominait. Les mêmes constats ont été faits dans le rapport d'enquêtes sur les périmètres de Tiaguiréré amont, Tiaguiréré aval, Say 1, Say extension, Lata et Daïbéri où une bonne majorité de producteurs ont opté pour la variété Alewa qui n'est pas homologuée (MAG et KFW, 2015b). Dans les études menées par Mossi Maïga (2009) sur les périmètres de Yelawani, Saga et Sébéri, les responsables des coopératives de ces périmètres ont aussi fait cas de la production de riz avec des variétés qui n'étaient pas toutes homologuées. Les mêmes pratiques sont observées par Sido et Mossi Maïga (2003) dans une étude qui a été menée sur les périmètres de Karma, Lata, Koutoukalé, et Toula toujours au Niger où se côtoyaient variétés locales et variétés homologuées. On peut donc noter que l'utilisation des variétés non homologuées est une pratique qui se généralise, de plus en plus, sur l'ensemble des périmètres rizicoles irrigués. Pourtant, ces périmètres sont régis par des règles strictes de mise en valeur. Ces règles, en effet, interdisent l'utilisation, par les producteurs, de toute variété non homologuée. Il faut, cependant, noter que ces pratiques ne sont pas spécifiques qu'aux seuls périmètres rizicoles nigériens. Un peu partout en Afrique, comme l'ont montré les études menées, par le CNI-Ben 2009 et 2010 au Burkina Faso, par Marissal en 2011 en Guinée, par Fall et Cisse en 2011dans les bassins de production de riz de Kayes au Sénégal et par Ly et al. en 2001 en Guinée, la règle faisant obligation de l'utilisation de variétés homologuées sur les périmètres rizicoles irriguées n'est pas respectée. Ces variétés dites communautaires ou traditionnelles se sont imposées sur la majorité des périmètres irrigués rizicoles. Et, partout, ce sont les mêmes raisons qui militent en leurs faveurs à savoir : les rendements relativement élevés, la résistance aux maladies et aux oiseaux, la résistance au déficit hydrique, le port de la plante, la couleur des grains, le pourcentage de brisure lors du décorticage et le goût du riz.

Dans notre étude, on constate que les semis dans les pépinières ont suivi les mêmes étapes sur les périmètres de Bonféba et de Toula. Cependant, les quantités de semence utilisées étaient loin de celles que l'encadrement technique a proposé pour le repiquage d'un hectare de riz et qui était de $50 \mathrm{~kg}$ (Ekoye $e t$ al., 1996). Cette valeur était de 1,44 fois supérieure à celle utilisée dans les cas de nos deux périmètres. Plusieurs études, aussi bien dans les périmètres irrigués au Niger que dans d'autres pays, ont montré aussi que les quantités de semences pour repiquer un hectare de riz étaient loin des normes préconisées. Sur les périmètres irrigués de Gaya amont, de Liboré et de Say 1 au Niger, les quantités de semences utilisées étaient respectivement de $60 \mathrm{~kg} \mathrm{ha}^{-1}, 75 \mathrm{~kg} \mathrm{ha}^{-1}$, et de $50 \mathrm{~kg} \mathrm{ha}^{-1}$. On notera que la norme était respectée sur le périmètre de Say 1 (MAG, KFW, 2015a). Sur d'autres périmètres les résultats des enquêtes qui avaient été menées ont montré que la quantité de semences qui était utilisée pour repiquer l'hectare de rizière était rarement conforme à la norme de $50 \mathrm{~kg}$ ha-1 qui était recommandée par l'encadrement technique (MAG et KFW (2015b). Sur les six périmètres (Say extension, Daïbéri, Lata, Tiaguiéré aval, Sébéri et N'dounga 1) qui étaient concernées, la quantité de semence a varié entre 28 et $61 \mathrm{~kg} \mathrm{ha}^{-1}$. Les valeurs plus faibles étaient enregistrées sur périmètre de Sébéri où des essais sur le système de riziculture intensive (SRI) étaient en cours. Le SRI, en effet, préconise des quantités de semences plus faibles; pas plus de $10 \mathrm{~kg} \mathrm{ha}^{-1}$ (Styger et Jenkings, 2014). L'ensemble de ces études ont montré que la quantité de semence qui devrait être semée en pépinière pour pouvoir repiquer un hectare de riz n'était pas respectée. Ceci serait dû au fait que le paquet technique, qui était préconisé pour la préparation des semences, n'était pas appliqué 
dans son ensemble. Il s'agit, en effet, de la prégermination ainsi que le traitement des semences qui sont, de plus en plus, ignorés par les producteurs sur les périmètres irrigués rizicoles. De même, la règle du semis collectif n'est plus très suivie. Sur plusieurs périmètres irrigués rizicoles, en effet, le semis est devenu individuel. Ce sont donc ces nouvelles pratiques (abandon de la prégermination des semences, non traitement des semences, pépinières individuelles) qui expliquent les quantités élevées de semence consacrées pour le repiquage d'un hectare (Mossi Maïga, 2009; MAG et KFW, 2015a, 2015b ).

Le repiquage est systématique sur les périmètres rizicoles irrigués au Niger. Sur nos deux périmètres, il se fait à 45-60 jours après le semis selon les saisons culturales. Dans notre étude, les producteurs semblaient dire que la densité de repiquage était élevée dans leurs parcelles de riz. Le non-respect des densités de semis proposées par l'encadrement technique ont été remarqué aussi sur plusieurs autres périmètres au Niger. Les rapports des études réalisées par MAG et KFW en 2015 sur une dizaine de périmètres rizicoles irrigués au Niger ont montré que les densités de repiquages, dont la norme est de 250000 plants par hectare, n'étaient pas respectées sur aucun des périmètres (MAG et KFW 2015a ; MAG et KFW, 2015b). Sido et Mossi Maïga (2003), dans des enquêtes agronomiques réalisées sur 5 périmètres irrigués rizicoles au Niger à savoir ceux de Saga, Karma, Lata, Toula et Koutoukalé, ont relevé que les densité de repiquage variaient entre 138000 et 218000 poquets par hectare soit $55 \%$ et $87 \%$ de la densité prescrites. Les détails par périmètre ont montré que sur le périmètre de Karma la densité moyenne de repiquage était de 1,33 fois supérieure à la densité recommandée. Sur le périmètre de Koutoukalé, elle était comprise entre 227500 poquets par hectare et 303300 poquets par hectare. Sur le périmètre de Saga, elle a varié entre 130000 et 218000 poquets par hectare.
La variabilité dans les pratiques agricoles se remarque surtout dans la fertilisation. Les doses ainsi que les périodes d'application étaient très variables selon les producteurs et le périmètre irrigué. Les quantités médianes d'engrais qui ont été apportées sur les périmètres de Bonféba et de Toula étaient respectivement de 1,2 fois et de 1,7 fois supérieures à la dose qui était proposée par l'encadrement technique (Sido et al., 2015). En effet, la Figure 1 montre qu'une majorité des producteurs ont apporté des quantités d'engrais bien au-delà de $400 \mathrm{~kg} \mathrm{ha}^{-1}$. Tout comme les résultats de notre étude, d'autres études menées aussi bien au Niger que dans d'autres pays sur les périmètres irrigués rizicoles ont montré qu'il y avait une très grande variabilité des quantités totales d'engrais apportés par les producteurs. Les résultats des études qui étaient menées au Niger par MAG et KFW en 2015 sur les périmètres de Sayextension, Daïbéri, Lata, Tiaguiréré aval, Say 1 , ont montré que les quantités moyennes totales d'engrais apportées par producteur étaient, respectivement, de 1,$5 ; 1,25 ; 1,375$; 1,5 , et 1,25 fois supérieures à la moyenne proposée (MAG et KFW 2015a ; MAG et KFW, 2015b).Ces quantités étaient identiques, à quelque différence près, à celles qui ont été observées sur les périmètres de Bonféba et de Toula. Sur le périmètre de Kafiguéla au Burkina Faso par contre, les études ont montré que les quantités d'engrais apportés étaient loin des doses recommandées. Elles n'étaient, en effet, que de 0,375 fois inférieure à la dose recommandée (CNI-B, 2009). On constate ainsi que sur les périmètres irrigués rizicoles, à côté des prescriptions en matière de fertilisation, il y a une large gamme de formules de fertilisation que les producteurs adoptent. Par ailleurs, ces quantités d'engrais sont apportés comme engrais de couverture alors que les prescriptions préconisent que les engrais composés (NPK) soient apportés comme fumure de fond.

Sur nos deux périmètres (Bonféba et Toula), les producteurs ont réparti les quantités 
d'engrais en trois apports comme nous montrent les Figures 2, 3 et 4 en se définissant comme référence le nombre de jours après le repiquage. Les quantités d'éléments fertilisants à savoir l'azote, le phosphore et le potassium, apportées par ces engrais sont montrées par les Tableaux 5, 6 et 7. Ces résultats ont montré aussi les écarts par rapport aux recommandations de l'encadrement technique local. Il faut, en effet, noter que l'encadrement proposait $400 \mathrm{~kg} \mathrm{ha}^{-1}$ d'engrais dont $200 \mathrm{~kg}$ ha $^{-1}$ d'engrais composé (15-15-15) comme engrais de fond au moment du repiquage et $200 \mathrm{~kg}$ ha-1 d'urée en engrais de couverture répartis en 2 apports dont $100 \mathrm{~kg} \mathrm{ha}^{-1} 14$ jours après le repiquage et $100 \mathrm{~kg} \mathrm{ha}^{-1} 35$ jours après le repiquage. Les prescriptions en elles-mêmes au Niger varient selon les sources et les services d'encadrement ou les projets de développement qui encadrent les producteurs. Dans le catalogue national des variétés de riz au Niger les quantités d'engrais proposées pour une meilleure valorisation des nouvelles variétés était de $500 \mathrm{~kg} \mathrm{ha}^{-1}$ dont $300 \mathrm{~kg}$ de NPK (15-15-15) en fumure de fond et $200 \mathrm{~kg}$ d'urée en fumure de couverture (MAG, 2012). Le projet GIPD (Gestion intégrée de la production et des déprédateurs) a proposé, pour tout type d'engrais confondu, la dose de $120 \mathrm{~kg} \mathrm{ha}^{-1}$ avec, cependant, un apport très important de fumure organique a été préconisée (MAG et KFW, 2015b).

La variabilité dans les quantités d'engrais s'observe également dans les prescriptions au niveau de différents pays dans la sous-région alors que les conditions de cultures sont pratiquement identiques. Au Mali les recommandations du projet amélioration de la productivité du riz en Afrique de l'Ouest était de 100 à $150 \mathrm{~kg} \mathrm{ha}^{-1}$ de phosphate d'ammoniaque (18-46-0) en fumure de fond et de 200 à $250 \mathrm{~kg} \mathrm{ha}^{-1}$ d'urée en deux apports en fumure de couverture(SPID, 2012). Au Sénégal, SAED (2009) a proposé $100 \mathrm{~kg} \mathrm{ha}^{-1}$ de phosphate d'ammoniaque (18-46-0) et 250 à $300 \mathrm{~kg} \mathrm{ha}^{-1}$ d'urée en trois apports. Au Burkina Faso l'encadrement technique a proposé $300 \mathrm{~kg} \mathrm{ha}^{-1}$ d'engrais NPK (15-1515 ) et $100 \mathrm{~kg} \mathrm{ha}^{-1}$ d'urée (CNI-B, 2009).

De cette étude, il se constate que les producteurs, dans leurs ensembles, adoptent des nouvelles pratiques, très souvent différentes des prescriptions techniques. Une enquête diagnostic menée par Mossi Maïga en 2016 apporte quelques réponses à cet état de fait. En effet, bien que disposant d'un conseiller technique permanent sur les périmètres, les producteurs ainsi que les responsables des coopératives estiment que le conseil technique est inexistant. Pour eux, le conseil manque de forme et n'a pas fondamentalement changé dans le fond en ce sens qu'il ne prend pas en compte les nouveaux défis qui se posent aux producteurs. Il doit, en effet, s'orienter vers une utilisation efficiente des intrants, une bonne gestion de l'eau. Comment transformer et commercialiser eux-mêmes les productions, comment avoir de nouveaux interlocuteurs pour la commercialisation des produits ou pour l'acquisition des intrants (Mossi Maïga, 2016). L'ensemble de ces préoccupations qui pose, outre le besoin d'un conseil efficace, pose aussi la question de la disponibilité et l'accessibilité des intrants agricoles. En effet, comment juger de l'efficacité d'un conseil agricole si les producteurs rencontrent des difficultés pour acquérir les intrants agricoles.

\section{CONCLUSION}

Cette étude effectuée dans les périmètres rizicoles de Bonféba et de Toula a donné un aperçu sur la très grande variabilité des pratiques culturales adoptées par les producteurs de riz au Niger. Malgré la présence quasi permanente d'un encadrement, elle met en évidence un déficit dans la qualité du conseil tel qu'il est actuellement diffusé sur les périmètres irrigués rizicoles. Il peine à toucher efficacement les producteurs. La différence entre la pratique sur le terrain et la norme prescrite nécessite que cette dernière soit évaluée régulièrement et réajustée en tenant compte des conditions, changeantes de 
l'environnement, en termes de disponibilité de main d'œuvre, des intrants agricoles en quantité et en qualité, de l'encadrement technique. Aussi, la délivrance du conseil technique doit être certainement revue ce qui permettra aux encadreurs de s'approprier de nouvelles techniques de l'information et de communication afin de toucher, efficacement, le plus grand nombre de producteurs.

\section{REMERCIEMENTS}

Les auteurs remercient très sincèrement l'Institut National de la Recherche Agronomique du Niger (INRAN) et AfricaRice pour avoir financé les travaux de recherche sans oublier les directeurs ainsi que les producteurs des périmètres irrigués.

\section{RÉFÉRENCES BIBLIOGRAPHIQUES}

AfricaRice, 2013. Methodology for diagnostic survey. Cotonou, AfricaRice. 9p.

CGERV, 2012. Diagnostic de la filière riz : campagne hivernage 2011-2012. Dakar, CGERV. 10p.

CNI-B, 2009. Diagnostic participatif du périmètre irrigué de Karfiguéla (province de la Comoé_Burkina Faso). Ouagadougou. IWMI/CILSS/USAID. 47p.

CNI-B, 2010. Diagnostic participatif et planification des actions du périmètre irrigué de Talembika. Ouagadougou. IWMI/ CILSS/USAID. 47p.

Ekoye, A., Tankari, D. et Schalbroek, 1996. Rapport annuel 1995 ONAHA.Niamey, MDR/HE. 30p.

Fall, A.A. et Cisse, A. 2011. Diagnostic de la filière riz dans les bassins de Kayes et analyse de situation socio-économique des membres de la plateforme rizicole. Dakar, VECO. 45p.

Ly, B.T., souare, D., Bah, E.S. et Bayo, L. 2001. Analyse-diagnostic des systèmes de production de riz en Guinée. Conakry. PASAL. 183p.
MAG, KFW, 2015a. Amélioration de la mise en valeur agricole des périmètres de Sayextension-Daïbéri-Lata-Tiaguiréré AvalTiaguiréré Amont-N'Dounga 1-Sébéri. Niamey, AHT Group/BETICO/GFA. 55p.

MAG, KFW. 2015b. Amelioration de la mise en valeur agricole des périmètres de Gaya Amont, Liboré et de Say 1.Niamey, AHT Group/BETICO/GFA. 55p

MAG, 2005. Stratégie nationale du développement de l'irrigation et des eaux de ruissellement (SNDI/CER). Niamey, Ministère du développement rural, $84 p$

MAG, 2012. Catalogue National des Espèces et Variétés Végétales (CNEV) : le Riz, Niamey, Ministère de l'agriculture. 39p.

Marissal, E. 2011. Analyse des facteurs de réussite de l'aménagement de bas-fonds en Guinée Forestière : Cas du bas fond de Soumanro appuyé par le projet ACORH. Mémoire de l'Ecole supérieure d'AgroDéveloppement International. CergyPontoise. 110p.

Mossi Maïga, I. 2009. Gestion collective des aménagements hydro-agricoles au Niger : gouvernance locale et mobilisation des ressources pour une mise en valeur viable, Doctorat d'Etudes Rurales, Universités de Toulouse le Mirail et Abdou Moumouni de Niamey, 298p.

Mossi Maïga, I. 2015. Le conseil agricole au Niger : Diagnostic du cas des perimeters irrigués. Niamey, GWI/IUCN. 23 p.

Poussin, J.C. et Boivin, P. 2002. Cultures irriguées et productions : Performances des systèmes rizicoles irrigués sahéliens. Cahiers Agricultures 11 : 65-73.

Poussin, J.C. 1994. Diagnostic sur les systèmes de riziculture irriguée dans la moyenne vallée aval du fleuve Sénégal. La Conduite du Champ Cultivé : Points de Vue d'Agronomes : Séminaire, Montpellier (FRA), 1994/09. ISBN 2-7099-1387-9

Sido, Y., Saminou, E. et Hassane A. 2015. Etat des lieux de la riziculture au Niger. Niamey, Ministère de l'Agriculture/ PPAAO-WAAPP-WAAPP. 92p. 
Sido, Y. et Mossi Maïga I. 2003. Enquête agronomique sur les périmètres irrigues. Niamey. Pafriz. 73p.

Styger, E. et Jenkins, D. 2014. Manuel technique sur le SRI en Afrique de l'Ouest :
Amélioration et mise à l'échelle du Système de Riziculture Intensive en Afrique de l'Ouest. Bamako, CORAF/WAAPP/CNSRiz/SRI-Rice. 